\title{
Changes in rubral multiunit activity after lesions in the interpositus nucleus of the cat
}

\author{
M. B. BROMBERG and S. GILMAN
}

Department of Neurology, The University of Michigan, Ann Arbor, Mich. 48109 (U.S.A.)

(Accepted March 23rd, 1978)

The initial effect of an acute lesion in the central nervous system is loss of neural function. With time, however, restorative processes, generally termed 'compensation', may result in some degree of recovery of neural function. The mechanisms involved in recovery may include morphological changes in synaptic connections, a process described as neuroplasticity. Direct evidence for neuroplasticity in the mature mammalian central nervous system has been presented (for recent reviews, see refs. 3-6).

Electrophysiological studies of the rubrospinal tract in the cat have shown evidence of neuroplasticity in which sprouting of axonal terminals (collateral sprouting) is thought to occur in the red nucleus following partially deafferenting lesions ${ }^{14}$. The demonstration of synaptic reorganization in this nucleus is based on evidence that the two major inputs to this nucleus, corticorubral and interpositorubral fibers, make synaptic connection with two separate portions of the membranes of rubral neurons. Tsukahara and co-workers ${ }^{12,13}$ showed in the intact cat that excitatory postsynaptic potentials (EPSPs) evoked in rubral neurons by stimulating the ipsilateral pericruciate cortex had relatively slow rise times, long decay phases, and low peak amplitudes. EPSPs evoked by stimulating the contralateral interpositus nucleus displayed more rapid rise times, shorter decay phases, and greater peak amplitudes. They attributed these differences to segregation of synaptic terminals on rubral neurons, cortical fibers on distal dendrites and interpositus fibers on proximal dendrites and somas. The reorganization of cortical synaptic contacts on rubral neurons after partial deafferentation was investigated in chronic cats with lesions of the contralateral interpositus nucleus ${ }^{14}$. They observed a progressive shortening of the rise times of cortically evoked EPSPs for 4-10 days after the lesions and no further change after longer intervals. These changes in cortically evoked EPSPs were thought to result from collateral sprouting of cortical fibers to innervate more proximal portions of rubral neurons.

If neuroplasticity participates in the recovery of motor function after a lesion, it must do so not only through changes in postsynaptic potentials, but also through changes in patterns of spike activity directed toward brain stem and spinal nuclei. It is not clear what effect changes in EPSP wave forms may have on rubral spike activity. We have investigated this issue by recording spontaneous multiunit spike activity in 
the red nucleus of awake cats for several weeks before and after lesions in the interpositus nucleus.

Five adult cats were prepared for chronic recording by implanting a fixed array of 1-3 stainless steel electrodes with tip impedances of $10-20 \mathrm{k} \Omega$ in the mesencephalon to record extracellular spike activity from a population of neurons. Electrodes were directed to one red nucleus and to areas of the mesencephalon 2-4 mm lateral to the red nucleus on the same side to determine whether the changes in activity observed after cerebellar lesions occurred elsewhere in the mesencephalon. Recordings were made with the animals awake. To ensure stable recording conditions the animals were confined in a nylon bag with only the head protruding and the bag was suspended free of any surface. Under these conditions, with no movement from the animal, the level of multiunit activity was stable. Burst of high amplitude activity occurred only when the animals made rapid movements and activity recorded during such movements was eliminated from the analysis. The amplitude of the multiunit activity was measured as the integral of the amplified, filtered ( $300 \mathrm{~Hz}-3 \mathrm{kHz}$ bandpass), fully rectified signal. In order to integrate activity for relatively long periods of time and retain adequate sensitivity, the integrator was reset periodically at an interval constant for each cat. The height of the integrator output voltage at reset, therefore, was proportional to the amplitude of the neural activity. Recordings were taken for several minutes per session and about 10 integrals were averaged to give data points. The experimental procedure was first to record activity 3 times weekly for several weeks to establish prelesion activity levels. Then an electrolytic lesion was made in the interpositus nucleus contralateral to the red nucleus under investigation and multiunit recordings were followed for another 4-8 weeks. In one animal, the ipsilateral pericruciate cortex was ablated subsequently by subpial aspiration. All recording electrode placements and lesions were verified histologically. The lesions in the interpositus nucleus included portions of the anterior and posterior divisions.

Activity recorded from the red nucleus (Fig. IA, C and E) was consistently greater in amplitude (mean for 3 animals $=51.8 \mu \mathrm{V}$ ) than that from the extrarubral sites (Fig. I B, D and F) (mean for the same 3 animals $=18.2 \mu \mathrm{V}$ ). The amplitudes were computed relative to the peak-to-peak voltages of a $1 \mathrm{kHz}$ sine wave after similar signal conditioning and integration. With a randomized block design analysis of variance, the activity recorded from the red nucleus was significantly different in amplitude from the activity recorded from the extrarubral sites $(F=67.16, \mathrm{df}=2,74, P<0.05)$.

After lesions in the contralateral interpositus nucleus, multiunit activity recorded from the red nucleus showed an initial decrease of amplitude and a subsequent, slow return, whereas activity recorded from extrarubral sites showed no appreciable change (Fig. 1A, C, E and G, compared to B, D and F). A paired $t$-test was used to assess the difference in amplitude of activity measured from the red nucleus before and the day after lesions in the interpositus nucleus. In each of the 4 animals, the difference between the last measurement prelesion and the first measurement postlesion was computed. The mean difference was $19.0 \mu \mathrm{V}$, a value significantly different from zero $(t=3.81$, $\mathrm{df}=3, P<0.05$ ). Regression analyses were performed to compare the effects of interpositus lesions on multiunit activity over time. During the prelesion period, the average 

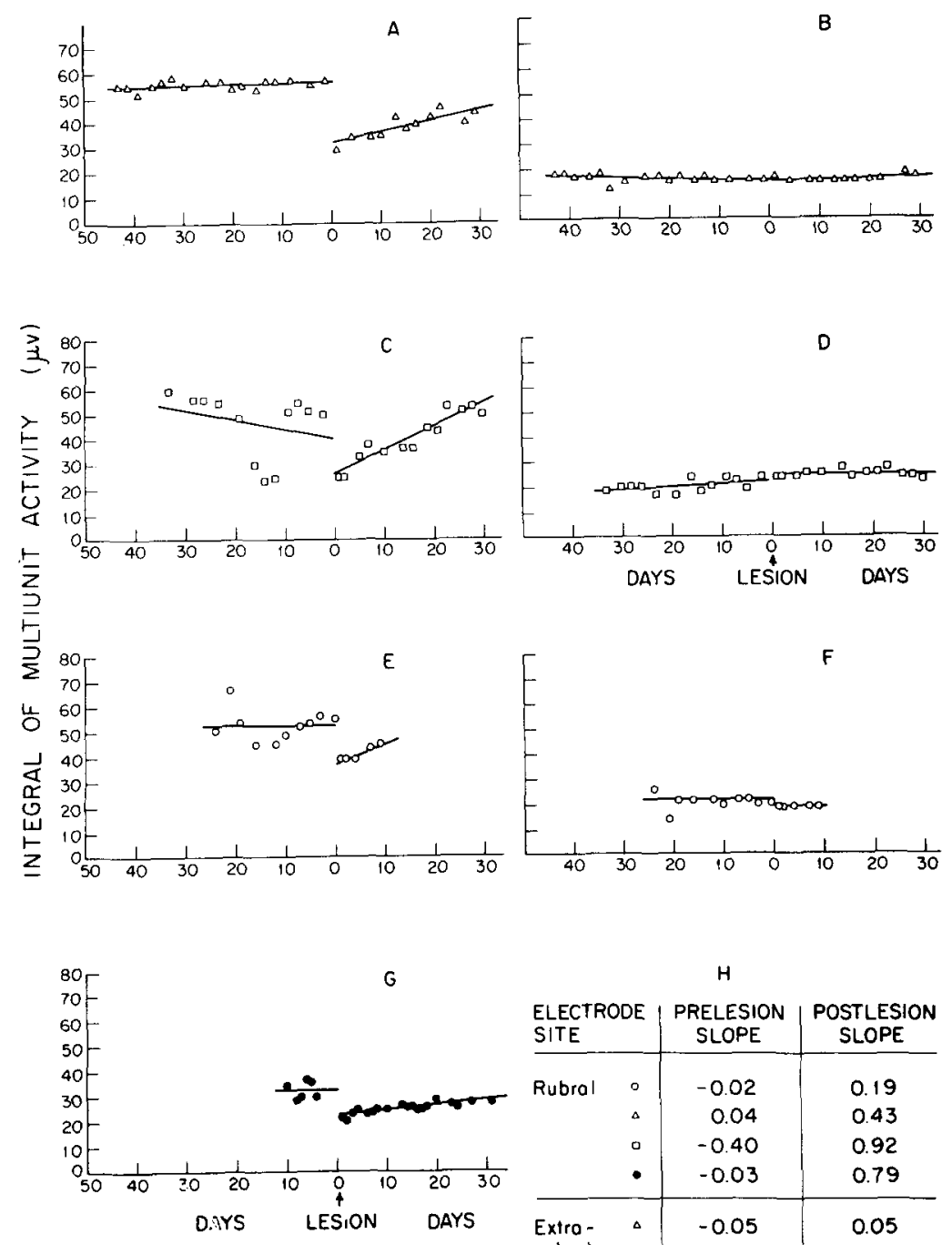

\begin{tabular}{lc|c|c}
\multicolumn{2}{l|}{$\begin{array}{c}\text { ELECTRODE } \\
\text { SITE }\end{array}$} & $\begin{array}{c}\text { PRELESION } \\
\text { SLOPE }\end{array}$ & $\begin{array}{c}\text { POSTLESION } \\
\text { SLOPE }\end{array}$ \\
\hline Rubral & $\circ$ & -0.02 & 0.19 \\
& $\Delta$ & 0.04 & 0.43 \\
& 0 & -0.40 & 0.92 \\
& - & -0.03 & 0.79 \\
\hline Extro- & $\triangle$ & -0.05 & 0.05 \\
rubrol & 0 & 0.10 & -0.01 \\
& - & -0.03 & 0.00
\end{tabular}

Fig. 1. Amplitude of multiunit activity recorded from fixed electrodes in mesencephalon before and after lesions in the contralateral interpositus nucleus. Abscissa, days on which recordings were made before and after lesions. Ordinate, integral of multiunit activity with respect to time $(\mu \mathrm{V})$. A, C, E and G: time course of activity changes from rubral recording sites, B, D and F: time course of activity recorded $2-3 \mathrm{~mm}$ lateral to red nucleus. $\mathrm{H}$ : table of slopes of regression lines fitted to data points. Each symbol represents data from different animal.

regression slopes did not differ significantly from zero for either rubral or extrarubral activity and, during the postlesion period, the regression slope of extrarubral activity likewise did not differ from zero $(t=1.27,0.14,0.70 ; \mathrm{df}=3,2,2$, respectively; $P>0.20$ for all 3 tests). The mean regression slope of rubral activity during the postlesion period, however, was positive and significantly greater than zero $(t=3.49$, df $=3, P<0.50)$, indicating that activity measured from this site increased in amplitude over time following the lesion (Fig. IH). 
In one animal the ipsilateral pericruciate cortex was ablated 6 weeks after the lesion in the contralateral interpositus nucleus to determine if the restoration of activity depended upon intact corticofugal fibers. After the ablation there was an immediate drop in amplitude of the activity, but a slow restoration of amplitude occurred over a 3 -week period. The slope of the regression line through the data points of the postlesion period is 0.21 , which is significantly different from zero $(t=2.54$, df $\ldots, P, P \quad 0.50)$.

The amplitude of multiunit activity recorded from fixed electrodes in awake. restrained cats has been shown to remain stable over long intervals of timel. In the present experiments, this was substantiated by regression lines for prelesion activity for each cat that were not significantly different from zero. In one animal (Fig. 1C) there was a decrease in amplitude of the activity without apparent cause, but this was followed by a return to a stable level before the lesion. It has been observed previously that multiunit activity recorded in the red nucleus is higher in amplitude than in nearby regions ${ }^{1}$. In the present study, the activity in extrarubral mesencephalic areas lateral to and at approximately the same depth as the red nucleus was unchanged in amplitude by lesions in the contralateral interpositus nucleus. The changes in activity in the red nucleus after lesions in the interpositus nucleus were characterized by an immediate reduction and slow but incomplete restoration of amplitude. The animals were observed for a limited time and it is not known whether a full restoration of amplitude may have occurred or how long it may have taken to occur. Tsukahara et al. ${ }^{14}$ showed that cortically evoked EPSP rise times change maximally by 10 days after lesions in the contralateral interpositus nucleus. The time course of the restoration of multiunit activity in the present study was longer than that observed by Tsukahara et al. ${ }^{14}$. One explanation of the difference is that collateral sprouting of cortical terminals may continue beyond 10 days, but only the initial sprouts making synaptic connections with the proximal portions of rubral neurons during the first 10 days are needed to produce an EPSP with minimal attenuation.

An additional lesion was made in the ipsilateral pericruciate region of cerebral cortex in one cat to determine whether cortical fibers are necessary for the restoration of multiunit activity after interpositus lesions. In the cat, nearly all corticorubral fibers originate from the ipsilateral pericruciate region ${ }^{11}$. The effect of the cortical ablation was an immediate reduction in amplitude of activity in the red nucleus, but no change in amplitude of activity in extrarubral positions. During 3 weeks of observation, a slow but incomplete recovery of rubral activity was observed. The reduction in the amplitude of spontaneous multiunit activity after both interpositus nucleus and cerebral cortex lesions supports the notion that cortical fibers may be at least partially responsible for the recovery of activity after the cerebellar lesions, possible through the process of collateral sprouting. The slow restoration of amplitude after both lesions may indicate collateral sprouting from other fiber systems.

Another process may contribute to the restoration of rubral multiunit activity after cerebellar and cerebral lesions. Destruction and subsequent removal of fibers and terminals following deafferentation would be expected to cause some shrinkage of the previously innervated tissue. This factor must be considered in any study of neuroplasticity based on changes in density of the measured parameters ${ }^{8}$. Shrinkage 
has been noted in several nuclei after partial deafferentation $2,7,9,10$, but has not been measured in the red nucleus after cerebellar or cerebral lesions. In the recordings described here, degeneration and shrinkage could bring closer to the recording electrodes neurons which never in fact regain their previous levels of activity. This movement of neurons relative to a fixed recording electrode could conceivably account for a restoration of multiunit activity amplitude in time. Changes in tissue impedance following loss of myelinated fibers also would be expected. The effects of cell density and tissue impedance on multiunit recording have been investigated, and no correlations were found between these parameters and the amplitude of unit activity ${ }^{1}$. Furthermore, in a study of neuroplasticity in the lateral geniculate nucleus, shrinkage has been noted without an increase in cell density? ${ }^{2}$. An estimate of the contribution of shrinkage to the level of multiunit activity described here must await a detailed determination of the time course of rubral shrinkage and change in cell density after cerebellar and cerebral lesions.

\section{Supported in part by USPHS Grant NS 10612.}

1 Buchwald, J. S. and Grover, F. S., Amplitudes of background fast activity characteristic of specific brain sites, J. Neurophysiol., 33 (1970) 148-159.

2 Goodman, D. C. and Horel, J. A., Sprouting of optic tract projections in the brainstem of the rat, J. comp. Neurol., 127 (1966) 71-88.

3 Guth, L., Axonal regeneration and functional plasticity in the central nervous system, Exp. Neurol., 45 (1974) $606-654$.

4 Guth, L., History of central nervous system regeneration research, Exp. Neurol., 48 (1975) 3-15.

5 Guth, L. and Windle, W. F., The enigma of central nervous regeneration, Exp. Neurol, 28, Suppl. $5(1970) 1-43$.

$6 \mathrm{Kerr}$, F. W. L., Structural and functional evidence of plasticity in the central nervous system, Exp. Neurol., 48 (1975) 16-31.

7 Liu, C. and Chambers, W. W., Intraspinal sprouting of dorsal root fibers, Arch. Neurol. Psychiat. (Chic.), 79 (1958) 46-61.

8 Lynch, G. and Cotman, C. W., The hippocampus as a model for studying anatomical plasticity in the adult brain. In R. L. Isaacson and K. H. Pribram (Eds.), The Hippocampus, Vol. 1: Structure and Development, Plenum Press, New York, 1975.

9 Lynch, G., Rose, G., Gall, C. and Cotman, C. W., The response of the dentate gyrus to partial deafferentation. In M. Santini (Ed.), Golgi Centennial Symposium, Proceedings, Raven Press, New York, 1975.

10 Raisman, G. and Field, P. M., A quantitative investigation of the development of collateral reinnervation after partial deafferentation of the septal nuclei, Brain Research, 50 (1973) 241-264.

11 Rinvik, E. and Walberg, F., Demonstration of a somatotopically arranged cortico-rubral projection in the cat. An experimental study with silver methods, J. comp. Neurol., 120 (1963) 293-407.

12 Toyama, K., Tsukahara, N., Kosaka, K. and Matsunami, K., Synaptic excitation of red nucleus neurons by fibers from interpositus nucleus, Exp. Brain Res., 11 (1970) 187-198.

13 Tsukahara, N. and Kosaka, K., The mode of cerebral excitation of red nucleus neurons, Exp. Brain Res., 5 (1968) 102-117.

14 Tsukahara, N., Hultborn, H., Murakami, F. and Fujito, Y., Electrophysiological study of formation of new synapses and collateral sprouting in red nucleus after partial denervation, $J$. Neurophysiol., 38 (1975) 1359-1372. 